\title{
Effect of Aerodynamic Configuration Parameters on Lateral-Directional Stability of Double-Swept Flying Wing Aircraft
}

Ya Lin Pan ${ }^{1, *}$, Jun Huang ${ }^{1}$

Pan YL (D) https://orcid.org/0000-0001-7446-114X

Huang J (DD https: / /orcid.org/0000-0002-2827-8990

\section{How to cite}

Pan YL; Huang J (2019) Effect of Aerodynamic Configuration Parameters on Lateral-Directional Stability of Double-Swept Flying Wing Aircraft. J Aerosp Technol Manag, 11: e3919. https://doi. org/10.5028/jatm.v11.1074

ABSTRACT: Poor lateral-directional stability due to the absence of vertical stabilizer is a great risk to the aircraft with flying wing layout. In this paper, an unmanned aerial vehicle with this kind of configuration is chosen as the research object. A threedimensional model of the unmanned aerial vehicle is established, and then the sensitivity analysis is performed to obtain the effects of main aerodynamic shape parameters on lateral-directional flying quality. The results show that the roll mode and spiral mode of the aircraft meet the requirements of Level 1 flying quality in MIL-F-8785C. But the Dutch roll mode is generally divergent, which means that the flying quality of the aircraft is unacceptable. Thus it can be seen that the Dutch roll mode is the key to the dynamic stability of the aircraft. Further studies show that increasing the value of wing aspect ratio or decreasing the values of dihedral angle and torsion angle are useful for improving the Dutch roll mode. It is valuable to reveal the influence mechanism of aerodynamic shape parameters on lateral-directional flying quality for the design of flying wing aircraft.

KEYWORDS: Dynamic stability, Flying wing, Sensitivity analysis, Dutch roll mode.

\section{INTRODUCTION}

Flying wing configuration is considered as an important development direction of military aircraft and civil aircraft due to its potential benefits over conventional configurations in stealth capability, aerodynamic performance, and structural efficiency (Zhou and Liu 2015). In recent years, flying wing configuration has been widely studied (Alsahlan and Rahulan 2017; Bolsunovsky et al. 2014; Pan et al. 2017), and it has been considered as an ideal configuration of the future unmanned aerial vehicles (UAV) (Petterson 2006).

However, there is a great risk in the stability and control for this kind of aircraft. The ideal flying-wing aircraft always combines wing and fuselage into an integral structure without horizontal or vertical stabilizers. The absence of vertical stabilizer leads to the deterioration of lateral-directional flying quality, and the yaw stiffness for this unconventional configuration is always neutral or slightly negative (Saeed et al. 2009; Bolsunovsky et al. 2001; Zhang et al. 2012b). Thus, the lateral-directional stability of such aircraft is often at a disadvantage, which poses a great challenge to the designers for this kind of aircraft. Referring to the ongoing programs for research and development, there are two approaches to improve the lateral-directional dynamic stability of flying wing aircraft. One of the methods is making a large winglet at the wingtip of the aircraft, which produces the same effect as the vertical stabilizer (Snyder and Weisshaar 2013). This method is always used in civil aircraft, such as referenced in Zhang et al. (2012a)

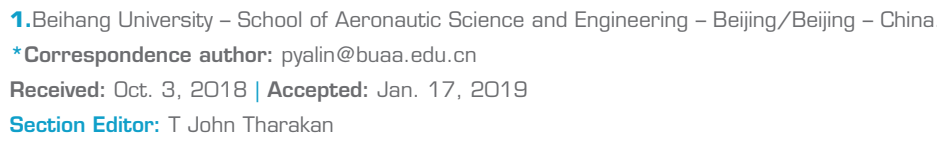


and Martinez-Val and Schoep (2000). The other method is deflecting a new type of aerodynamic control surfaces automatically to generate a restoring moment. This method is usually adopted in military aircraft. For instance, the drag rudders of B-2 stealth bomber, the crow mixing differential flaps of X-45 unmanned aerial vehicle (UAV), and a combination of spoilers and trailing edge control surfaces of X-47 UAV are all used for realizing this function (Grellmann 1990; Davidson 2004; Whittenbury 2011). Although both the methods can provide effective stability for flying wing aircraft, they have negative impacts on the radar crosssection, structural weight and aerodynamic efficiency of the aircraft. The early exploration of flying wing aircraft provides another approach to improving the flying quality of such aircraft. The Ho. 229 achieved an acceptable lateral-directional flying quality by using an appropriate sweepback angle and taper ratio (Song et al. 2014). It illustrates that lateral-directional dynamic stability can be achieved at an acceptable flying quality by optimizing the aerodynamic shape parameters of the aircraft.

In this paper, a double-swept flying wing aircraft is taken as an object for carrying out the sensitivity analysis of lateraldirectional stability. First, the three-dimensional model of the aircraft is established. The configuration used as a basis is shown in Fig. 1. The main design parameters of the presented UAV include wingspan $40 \mathrm{~m}$, wing area $157.3 \mathrm{~m}^{2}$, and flight weight $14800 \mathrm{~kg}$. Then, the impact of main aerodynamic shape parameters on the lateral-directional stability is investigated. The vortex lattice method is adopted to calculate the related aerodynamic parameters of the UAV. Once these parameters are obtained the small disturbance linear equation based on the UAV is used to get the assessment parameters of lateral-directional flying quality. It is useful to research the sensitivity of the impact of aerodynamic shape parameters on dynamic stability of flying-wing aircraft. This paper aims to reveal the influence mechanism of aerodynamic configuration parameters on the stability of the aircraft, and to provide a theoretical basis for the conceptual design and the flight control system design of the aircraft.

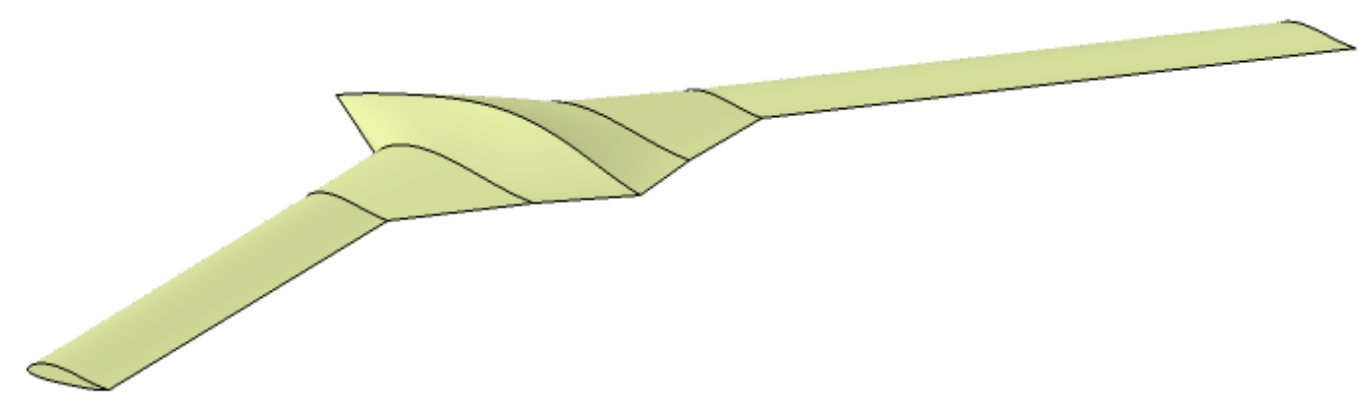

Figure 1. CAD model of flying wing aircraft.

\section{RESEARCH OBJECT}

A kind of double-swept flying wing UAV is taken as the research object. The planform of the blend-wing-body aircraft is displayed in Fig. 2. The initial design parameters of the UAV are calculated according to traditional design method. This configuration can be considered as a combined wing made up by three segments. The main outline parameters include the reference area of aircraft $(S)$, wing aspect ratio $(A r)$, root chord length of inner wing $(c 1)$, root chord length of outer wing $(c 2)$, semi span of inner wing (b1), leading edge swept angle of inner wing $(\Lambda 1)$, leading edge swept angle of outer wing $(\Lambda 2)$, dihedral angle $(\Gamma)$, and angle of torsion $(\Phi)$. These main parameters are independent to each other. The other planform parameters, such as semi span of Segment 2 (b2), semi span of Segment 3 (b3), and chord length of Segment 3 (c3 and $c 4$ ), shown in Fig. 2, can be derived from the main outline parameters.

It is necessary to obtain the aerodynamic performance parameters to evaluate flying quality. The variation of aerodynamic shape parameters will lead to the changes in aerodynamic force and aerodynamic moment of the aircraft. In the sensitivity analysis 


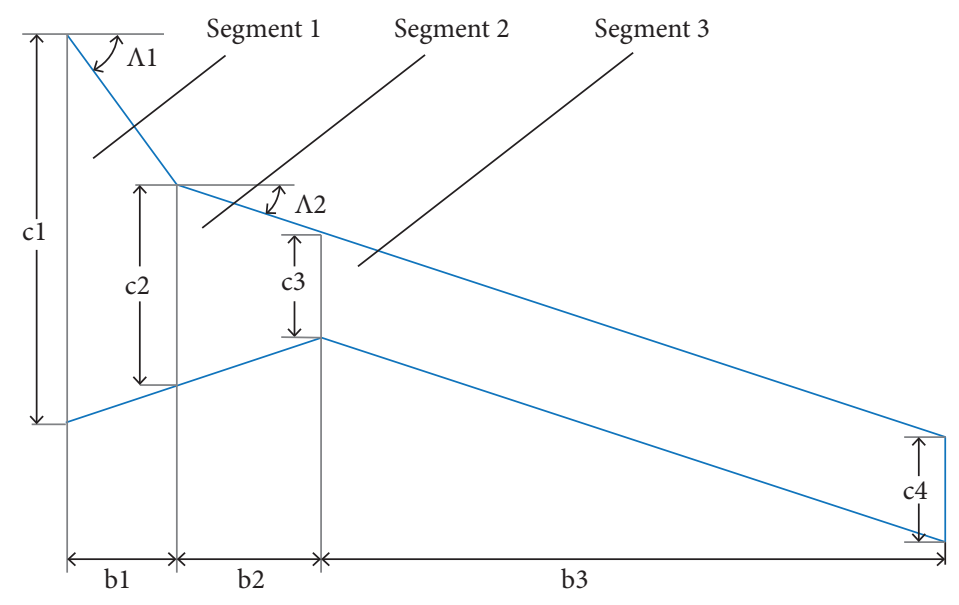

Figure 2. Half of flying wing aircraft platform.

of stability, the aerodynamic performance corresponding to a large number of aircrafts with different outline parameters should be calculated. The vortex lattice method is adopted to get these aerodynamic coefficients and stability derivatives. In the method, aerodynamic performance is predicted by solving the potential flow equation instead of solving Navier-Stokes equation. The potential flow equation is a simplification of Navier-Stokes equation under the assumption of inviscid and irrotational flow. Thus the method has a higher computation rate than CFD codes. The limitations of this method are that it can only predict the aerodynamic performance of aircraft at small angle of attack, and it cannot calculate the frictional drag. In the paper, the component buildup method is used to obtain the zero lift drag coefficient as a subsidiary part of the method. Detailed calculation procedures of this method have been given in Melin (2000) and Song et al. (2014).

The accuracy of this method has been widely validated (Song et al. 2014; Mialon et al. 2011). In this paper, a generic UAV configuration named SACCON (Stability And Control Configuration) is used to assess the ability of the suggested method for predicting aerodynamic characteristics of flying wing aircraft. Detailed description of the configuration and its design process can be found in Liersch and Huber (2014) and Vicroy et al. (2010). The flow conditions are set with $M a=0.15$ and $R e=1.60 \times 10^{6}$, which are consistent with the wind tunnel experiment. Figure 3 shows a comparison of aerodynamic force and moment coefficients of the standard model. It can be seen that the calculation results have good agreement with the wind tunnel data or the reference data (Cummings et al. 2016; 2010), except for the pitching moment coefficient. As Fig. 3 shows, the pitching moment coefficient curve has a slight deviation, especially for higher angles of attack. The deviation only means a small discrepancy in the position of the neutral point because it is located very close to the moment reference point. Compared to the vortex lattice method results and the experimental data, the lift curve has a slightly higher slope and a minimal shift in the zero-lift angle. By comparison with the lift curve in Cummings et al. (2016), the error caused by this method is acceptable in conceptual design of aircraft. The side force coefficients and the rolling and yawing moment coefficients are all zero, which are reasonable for the symmetrical geometry under symmetrical flow conditions. The drag curve of the calculation result differs significantly from the experimental results. That is because of the influence coming from the sting of the wind-tunnel model, which has been analyzed in Schütte et al. (2014). The aerodynamic parameters obtained by solving Navier-Stokes equation could be closer to the wind tunnel data than the presented method, but the improvements cannot have a significant impact on the flying quality of aircraft. Due to a great number of designs should be judged and weighed in conceptual design of aircraft, the computational efficiency of aerodynamic performance is as important as its calculation accuracy. In the following section, the flying qualities of aircraft under level flight state are calculated, which maintains a low angle of attack. This made the method a preferable choice for efficiently, obtaining a great number of calculations required in sensitivity analysis. 

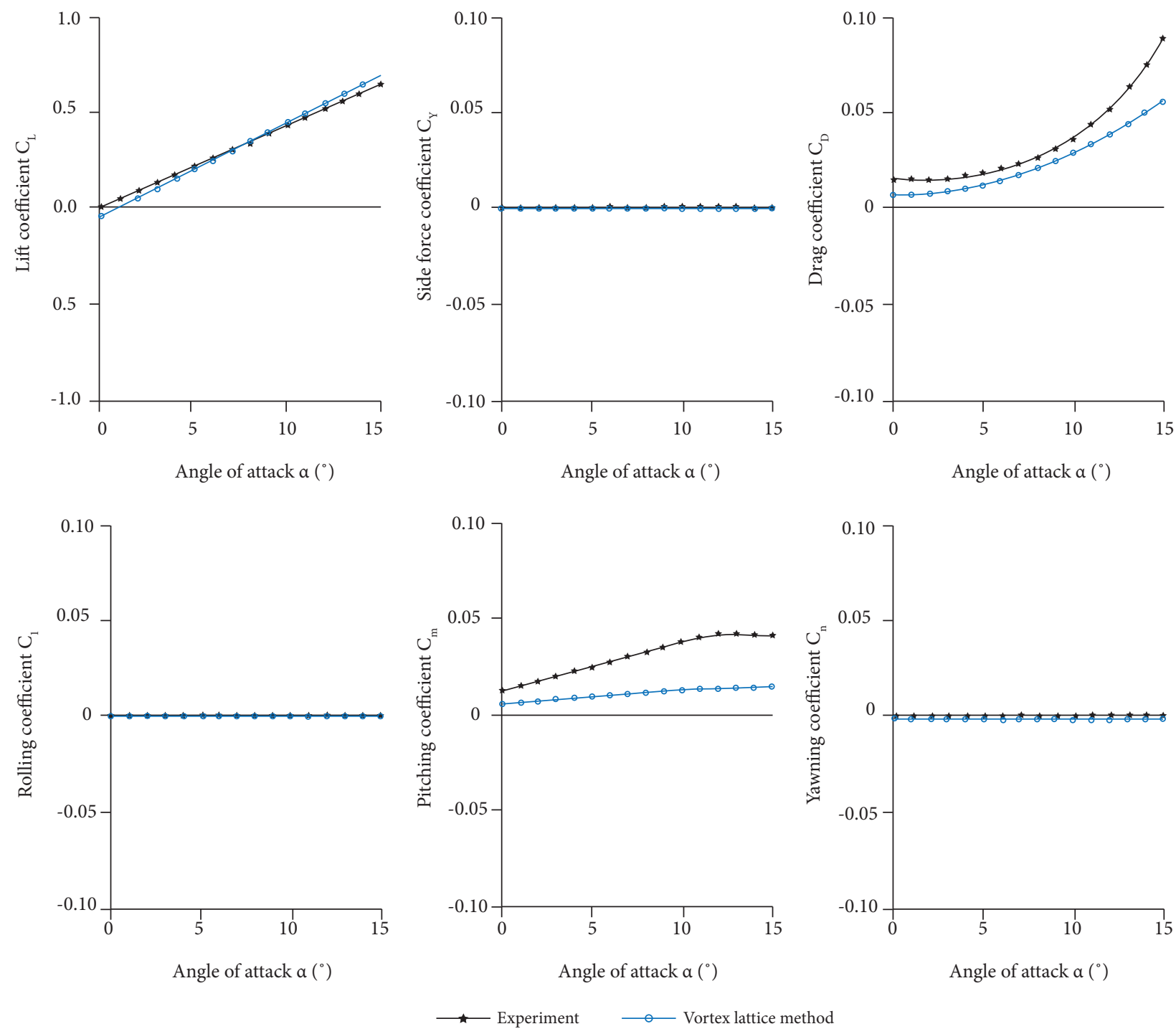

Figure 3. Comparison of coefficients of the reference model.

\section{CALCULATION OF PARAMETERS FOR STABILITY ASSESSMENT}

This paper only researches the lateral-directional flying quality of the presented UAV marginally affected by the position of the center of gravity. The assessment criteria of lateral-directional stability are always related to the characteristic parameters of the motion modes. The lateral-directional motion modes generally contain the roll mode, Dutch roll mode, and spiral mode. It is reasonable to give up estimating the lateral-directional static stability derivative and set the dynamic stability as the criterion to guarantee the flying quality in aircraft conceptual design (Song et al. 2015).

The dynamic property of an aircraft is determined by the eigenvalues of lateral-directional motion modes. The eigenvalues of the motion modes could be calculated by the matrix, which is constructed by bringing lateral-directional aerodynamic derivatives and inertial parameters into the lateral-directional small disturbance linear equations of the UAV. The matrix $A_{\text {lat }}$ (Fang et al. 2005) is presented as (Eq. 1): 


$$
A_{\text {lat }}=\left[\begin{array}{cccc}
\overline{Y_{\beta}} & \alpha_{*}+\overline{Y_{p}} & \overline{Y_{r}}-1 & g \cos \theta_{*} / V_{*} \\
\overline{L_{\beta}} & \overline{L_{p}} & \overline{L_{r}} & 0 \\
\overline{N_{\beta}} & \overline{N_{p}} & \overline{N_{r}} & 0 \\
0 & 1 & \tan \theta_{*} & 0
\end{array}\right\rfloor
$$

where: $\quad \overline{Y_{\beta}}=\frac{C_{y \beta} q_{*} S}{m V_{*}}, \quad \overline{Y_{p}}=\frac{C_{y p} q_{*} S}{m V_{*}} \cdot \frac{b}{2 V_{*}}, \quad \overline{Y_{r}}=\frac{C_{y r} q_{*} S}{m V_{*}} \cdot \frac{b}{2 V_{*}}, \quad N_{\beta}=C_{n \beta} q_{*} S b, N_{p}=C_{n p} q_{*} S b \frac{b}{2 V_{*}}$,

$$
\begin{aligned}
& N_{r}=C_{n r} q_{*} S b \frac{b}{2 V_{*}}, \quad L_{\beta}=C_{l \beta} q_{*} S b \quad, \quad L_{p}=C_{l p} q_{*} S b \frac{b}{2 V_{*}}, L_{r}=C_{l r} q_{*} S b \frac{b}{2 V_{*}}, \overline{L_{i}}=\frac{L_{i}+\left(I_{z x} / I_{z}\right) N_{i}}{I_{x}-I_{z x}^{2} / I_{z}}, \\
& \overline{N_{i}}=\frac{N_{i}+\left(I_{z x} / I_{z}\right) L_{i}}{I_{z}-I_{z x}^{2} / I_{x}} ; C_{l \beta}, C_{n \beta}, \text { and } C_{y \beta}
\end{aligned}
$$

represent the aircraft sideslip derivatives; $C_{l p}, C_{n p}$, and $C_{y p}$ represent the aircraft damping in roll derivatives; $C_{l r}, C_{n r}$, and $C_{y r}$ represent the aircraft damping in yaw derivatives; $I_{x}, I_{z}$, and $I_{z x}$ represent the aircraft moments of inertia $\left(\mathrm{kg} \cdot \mathrm{m}^{2}\right) ; b$ represents the span of aircraft $(\mathrm{m}) ; S$ represents the reference area of aircraft $\left(\mathrm{m}^{2}\right) ; q_{*}$ represents the dynamic pressure $\left(\mathrm{N} / \mathrm{m}^{2}\right) ; V_{*}$ represents the flight speed $(\mathrm{m} / \mathrm{s})$; and $m$ represents the mass of the aircraft $(\mathrm{kg})$.

Normally, five parameters directly related to the eigenvalues are set as the final evaluation standard of lateral-directional flying quality. These parameters typically include the time constant $(c)$ of roll mode, the time to double the amplitude $\left(t_{2}\right)$ of spiral mode, the natural frequency $\left(\omega_{n}\right)$ and damping ratio $(\xi)$ of Dutch roll mode and their product $\left(\xi \cdot \omega_{n}\right)$ in MIL-F-8785C (Department of Defense 1980). For Dutch roll mode, only $\xi$ is calculated to estimate the convergence property of the mode in this paper. The presented parameters for evaluating the lateral-directional dynamic stability can be calculated by the eigenvalues of the state matrix. A necessary and sufficient condition for the lateral-directional stability of an aircraft is that the determination parameters meet the requirements of flying quality.

The eigenvalues of the lateral-directional small disturbance equation are mainly determined by the lateral-directional aerodynamic derivatives of an aircraft. For flying wing aircraft, the variation in the shape parameters will change the aerodynamic derivatives, thus affecting the lateral-directional dynamic stability of the configuration. In order to get the property parameters of lateral-directional dynamic stability, the following steps should be executed. First, the level-flight angle of attack (AOA) at a certain speed for a typical level flight is computed by interpolating lift coefficients, which are obtained by the vortex lattice method. Then, the vortex lattice method is adopted again to calculate the stability derivatives under the level-flight angle of attack and the moments of inertia are calculated by building main components of the UAV. Finally, these lateral-directional aerodynamic derivatives and the inertia parameters of the aircraft are substituted into the state matrix, which is based on the lateral-directional small disturbance linear equations, to obtain the eigenvalues of each motion mode and the assessment parameters of lateral-directional dynamic stability.

\section{DYNAMIC STABILITY SENSITIVITY ANALYSIS}

To investigate the influence of main aerodynamic shape parameters on the lateral-directional stability, the reference area of aircraft $(S)$, root chord length of inner wing $(c 1)$, root chord length of outer wing $(c 2)$ and semi span of inner wing $(b 1)$ are defined as the design parameters, which are invariable throughout the research. The values of the design parameters are shown in Table 1. The parameters such as the wing aspect ratio $(A r)$, leading edge swept angle of inner wing $(\Lambda 1)$, leading edge swept angle of outer wing $(\Lambda 2)$, dihedral angle $(\Gamma)$, and angle of torsion $(\Phi)$ are set as the design variables, which vary in a certain range. The initial values and the range of these parameters are shown in Table 2. In this research, when one of the design variables changes 
the other parameters are invariable. The flight conditions are set as $H=1600 \mathrm{~mm}$ and $V=206 \mathrm{~m} / \mathrm{s}$. According to the presented steps, the vortex lattice method is adopted to obtain the aerodynamic derivatives and lateral-directional small disturbance linear equations are used to get the eigenvalues of lateral-directional motion modes.

Table 1. Values of the design parameters.

\begin{tabular}{|c|c|}
\hline Name & Value \\
\hline$S$ & $157.3 \mathrm{~m}^{2}$ \\
\hline$b 1$ & $2.5 \mathrm{~m}$ \\
$c 1$ & $11.2 \mathrm{~m}$ \\
$c 2$ & $5.8 \mathrm{~m}$ \\
\hline
\end{tabular}

Table 2. Values of the design variables.

\begin{tabular}{|c|c|c|c|c|}
\hline Parameters & Original & Low bound & Up bound & Step \\
\hline Ar & 10.2 & 7.2 & 17.2 & 1.0 \\
\hline$\Lambda 1$ & $60.0^{\circ}$ & $55^{\circ}$ & $65^{\circ}$ & $1.0^{\circ}$ \\
\hline$\Lambda 2$ & $22.5^{\circ}$ & $20.5^{\circ}$ & $30.5^{\circ}$ & $1.0^{\circ}$ \\
\hline$\Phi$ & $0.0^{\circ}$ & $-5.0^{\circ}$ & $5.0^{\circ}$ & $1.0^{\circ}$ \\
\hline$\Gamma$ & $0.0^{\circ}$ & $-5.0^{\circ}$ & $5.0^{\circ}$ & $1.0^{\circ}$ \\
\hline
\end{tabular}

\section{IMPACT OF AR ON DYNAMIC STABILITY}

Table 3 and Fig. 4 show how wing aspect ratio affects the lateral-directional dynamic stability. The shadow part in Table 3 is the flying quality assessment parameters of the initial design. It can be seen from the table that the level-flight angle of attack decreases with the increases of the aspect ratio, indicating that the lift coefficient of the aircraft is improved. The time constant (Tr) of roll mode decreases significantly with the increases of the aspect ratio, suggesting that the convergence rate of the rolling mode increases gradually. It is clear that the damping ratios of all designs are negative in the range of $A r$. In other words, the Dutch roll mode is divergent, and the dynamic stability of this design does not meet the requirements of flying quality. Compared with the Dutch roll mode, the spiral mode has a better flying quality. As shown in Fig. 4, the roll mode and spiral mode meet the requirements of Level 1 flying quality in MIL-8787C ( $\left.\operatorname{Tr} \leq 1.0, t_{2} \geq 20\right)$, and $A r$ has little effect on the spiral mode.

Table 3. Influence of Ar on lateral-directional stability.

\begin{tabular}{|c|c|c|c|c|c|}
\hline \multirow{2}{*}{$\begin{array}{c}\text { Values } \\
\text { (Ar] }\end{array}$} & \multirow{2}{*}{ AOA (deg] } & Roll mode & \multicolumn{2}{|c|}{ Dutch roll mode } & \multicolumn{1}{c|}{ Spiral mode } \\
\cline { 2 - 6 } & & $\operatorname{Tr}[\mathbf{s}]$ & Eigenvalue & $\xi$ & 231.9263 \\
\hline 7.2 & 3.0948 & 1.0645 & $0.0423 \pm 0.4160 \mathrm{i}$ & -0.1012 & 231.9015 \\
\hline 8.2 & 3.0080 & 0.9726 & $0.0335 \pm 0.3984 \mathrm{i}$ & -0.0838 & 235.3666 \\
\hline 9.2 & 2.9458 & 0.8949 & $0.0269 \pm 0.3845 \mathrm{i}$ & -0.0699 & 239.4916 \\
\hline 10.2 & 2.8994 & 0.8291 & $0.0221 \pm 0.3734 \mathrm{i}$ & -0.0591 & 240.2310 \\
\hline 11.2 & 2.8639 & 0.7734 & $0.0183 \pm 0.3636 \mathrm{i}$ & -0.0503 & 244.7805 \\
\hline 12.2 & 2.8376 & 0.7258 & $0.0153 \pm 0.3553 \mathrm{i}$ & -0.0430 & 243.3222 \\
\hline 13.2 & 2.8163 & 0.6845 & $0.0128 \pm 0.3510 \mathrm{i}$ & -0.0364 & 248.4610 \\
\hline 14.2 & 2.7999 & 0.6487 & $0.0107 \pm 0.3440 \mathrm{i}$ & -0.0310 & 243.3563 \\
\hline 15.2 & 2.7864 & 0.6174 & $0.0090 \pm 0.3413 \mathrm{i}$ & -0.0265 & 249.1783 \\
\hline 16.2 & 2.7758 & 0.5894 & $0.0076 \pm 0.3359 \mathrm{i}$ & -0.0226 & -0.0187 \\
\hline 17.2 & 2.7678 & 0.5648 & $0.0062 \pm 0.3336 \mathrm{i}$ & & 247.8594 \\
\hline
\end{tabular}



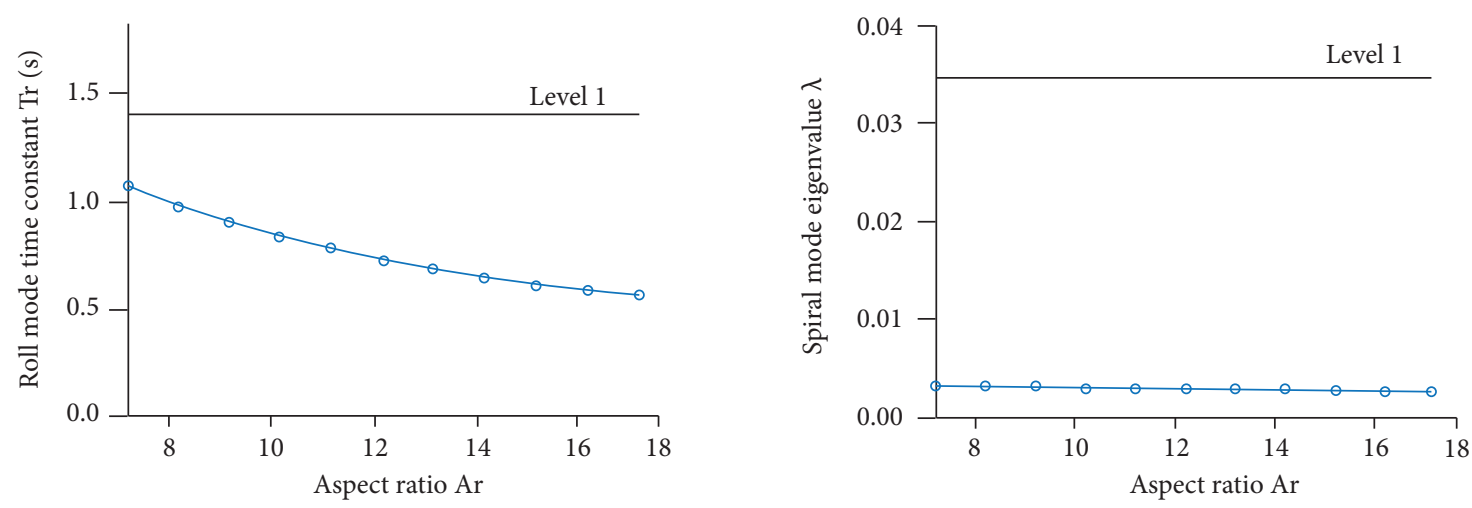

Figure 4. Influence of Ar on roll mode and spiral mode.

\section{IMPACT OF $\Lambda 1$ ON DYNAMIC STABILITY}

The impact of the leading edge swept angle of inner wing $(\Lambda 1)$ is presented in Table 4 and Fig. 5 . The calculation results show that the level-flight angle of attack decreases slightly with the increases of $\Lambda 1$. The reason for this case is that the semi span of Segment $2\left(b_{2}\right)$ increases with $\Lambda 1$, and the Segment 2 produces a greater lift to make up for the lift decrease of the inner wing. $\Lambda 1$ has little influence on the assessment parameters of lateral-directional dynamic stability. Although the roll mode and the spiral model meet the requirements of Level 1 flying quality, the lateral-directional dynamic stability of these designs is not acceptable due to the divergence of the Dutch roll mode. For this reason, great attention should be paid to the impact of the leading edge swept angle of inner wing on other performances in conceptual design of such aircraft.

Table 4. Influence of $\Lambda 1$ on lateral-directional stability.

\begin{tabular}{|c|c|c|c|c|c|}
\hline \multirow{2}{*}{$\begin{array}{l}\text { Values } \\
\text { [11] }\end{array}$} & \multirow{2}{*}{ AOA (deg) } & \multirow{2}{*}{$\frac{\text { Roll mode }}{\operatorname{Tr} \text { [s] }}$} & \multicolumn{2}{|c|}{ Dutch roll mode } & \multirow{2}{*}{$\frac{\text { Spiral mode }}{\text { t2 [s] }}$} \\
\hline & & & Eigenvalue & $\xi$ & \\
\hline 55 & 2.9107 & 0.8542 & $0.0227 \pm 0.3651 \mathrm{i}$ & -0.0621 & 245.5651 \\
\hline 56 & 2.9082 & 0.8508 & $0.0226 \pm 0.3657 \mathrm{i}$ & -0.0618 & 245.0561 \\
\hline 57 & 2.9069 & 0.8468 & $0.0226 \pm 0.3666 \mathrm{i}$ & -0.0614 & 244.4233 \\
\hline 58 & 2.9043 & 0.8420 & $0.0225 \pm 0.3677 \mathrm{i}$ & -0.0611 & 243.7896 \\
\hline 59 & 2.9019 & 0.8362 & $0.0224 \pm 0.3691 \mathrm{i}$ & -0.0606 & 243.0112 \\
\hline 60 & 2.8994 & 0.8291 & $0.0221 \pm 0.3734 \mathrm{i}$ & -0.0591 & 239.4916 \\
\hline 61 & 2.8957 & 0.8201 & $0.0218 \pm 0.3750 \mathrm{i}$ & -0.0580 & 238.1596 \\
\hline 62 & 2.8920 & 0.8081 & $0.0214 \pm 0.3771 \mathrm{i}$ & -0.0567 & 236.2344 \\
\hline 63 & 2.8858 & 0.7917 & $0.0206 \pm 0.3822 \mathrm{i}$ & -0.0539 & 227.9229 \\
\hline 64 & 2.8772 & 0.7672 & $0.0196 \pm 0.3857 \mathrm{i}$ & -0.0506 & 224.0075 \\
\hline 65 & 2.8638 & 0.7275 & $0.0176 \pm 0.3946 \mathrm{i}$ & -0.0446 & 212.4909 \\
\hline
\end{tabular}

\section{IMPACT OF $\Lambda 2$ ON DYNAMIC STABILITY}

The variation of the stability characteristics relevant to the leading edge swept angle of outer wing $(\Lambda 2)$ is displayed in Table 5 and Fig. 6 . Similar to $\Lambda 1, \Lambda 2$ has little impact on the assessment parameters of lateral-directional flying quality. Different from $\Lambda 1$, the trend of the time constant is increscent with the increasing of $\Lambda 2$. However, the difference does not improve the 
flying quality level of these modes. The time constant (Tr) of the roll mode and the time to double the amplitude $\left(t_{2}\right)$ of the spiral mode meet the requirements of Level 1 flying quality. But the Dutch roll mode is divergent in the whole range of $\Lambda 2$.
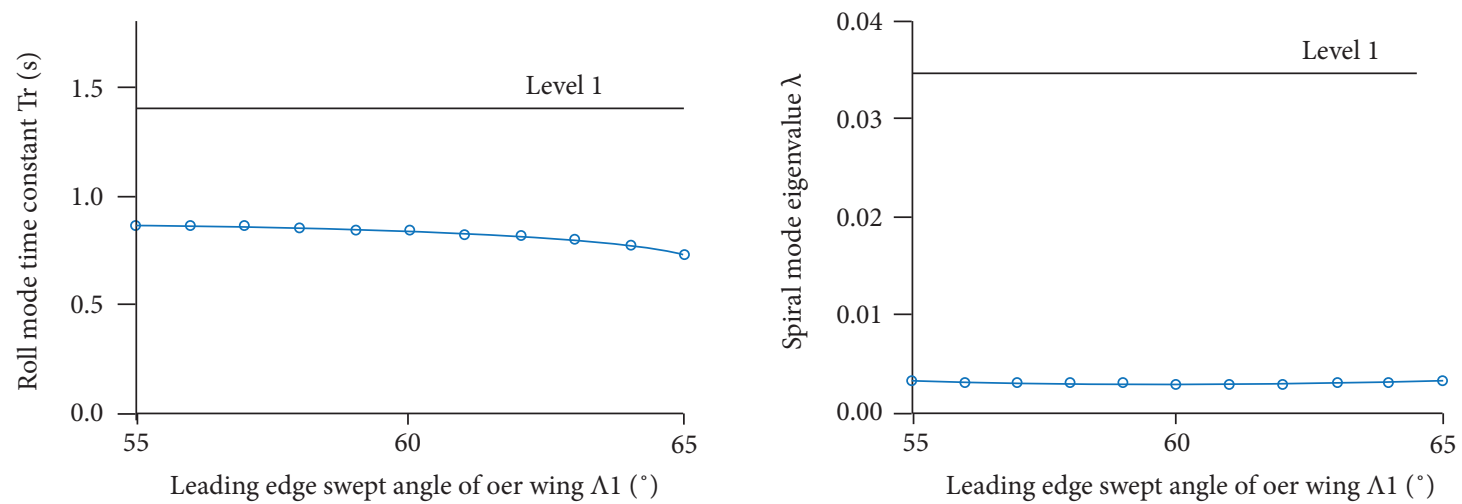

Figure 5. The influence of $\Lambda 1$ on roll mode and spiral model.

Table 5. Influence of $\Lambda 2$ on lateral-directional stability.

\begin{tabular}{|c|c|c|c|c|c|}
\hline \multirow{2}{*}{ 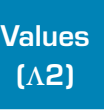 } & \multirow{2}{*}{ AOA [deg] } & \multirow{2}{*}{$\begin{array}{c}\text { Roll mode } \\
\operatorname{Tr}[\mathrm{s}]\end{array}$} & \multicolumn{2}{|c|}{ Dutch roll mode } & \multirow{2}{*}{$\frac{\text { Spiral mode }}{\text { t2 [s] }}$} \\
\hline & & & Eigenvalue & $\xi$ & \\
\hline 20.5 & 2.8712 & 0.8218 & $0.0187 \pm 0.3546 \mathrm{i}$ & -0.0526 & 241.0830 \\
\hline 21.5 & 2.8846 & 0.8255 & $0.0203 \pm 0.3654 \mathrm{i}$ & -0.0555 & 237.3261 \\
\hline 22.5 & 2.8994 & 0.8291 & $0.0221 \pm 0.3734 \mathrm{i}$ & -0.0591 & 239.4916 \\
\hline 23.5 & 2.9143 & 0.8330 & $0.0239 \pm 0.3809 \mathrm{i}$ & -0.0626 & 237.7733 \\
\hline 24.5 & 2.9306 & 0.8365 & $0.0258 \pm 0.3893 \mathrm{i}$ & -0.0662 & 239.6691 \\
\hline 25.5 & 2.9484 & 0.8401 & $0.0278 \pm 0.3974 \mathrm{i}$ & -0.0698 & 241.1605 \\
\hline 26.5 & 2.9676 & 0.8438 & $0.0298 \pm 0.4055 i$ & -0.0733 & 242.3603 \\
\hline 27.5 & 2.9870 & 0.8473 & $0.0320 \pm 0.4142 \mathrm{i}$ & -0.0770 & 243.6518 \\
\hline 28.5 & 3.0080 & 0.8510 & $0.0342 \pm 0.4252 \mathrm{i}$ & -0.0801 & 239.4537 \\
\hline 29.5 & 3.0307 & 0.8546 & $0.0365 \pm 0.4338 \mathrm{i}$ & -0.0839 & 240.2441 \\
\hline 30.5 & 3.0536 & 0.8583 & $0.0389 \pm 0.4426 \mathrm{i}$ & -0.0876 & 240.9533 \\
\hline
\end{tabular}
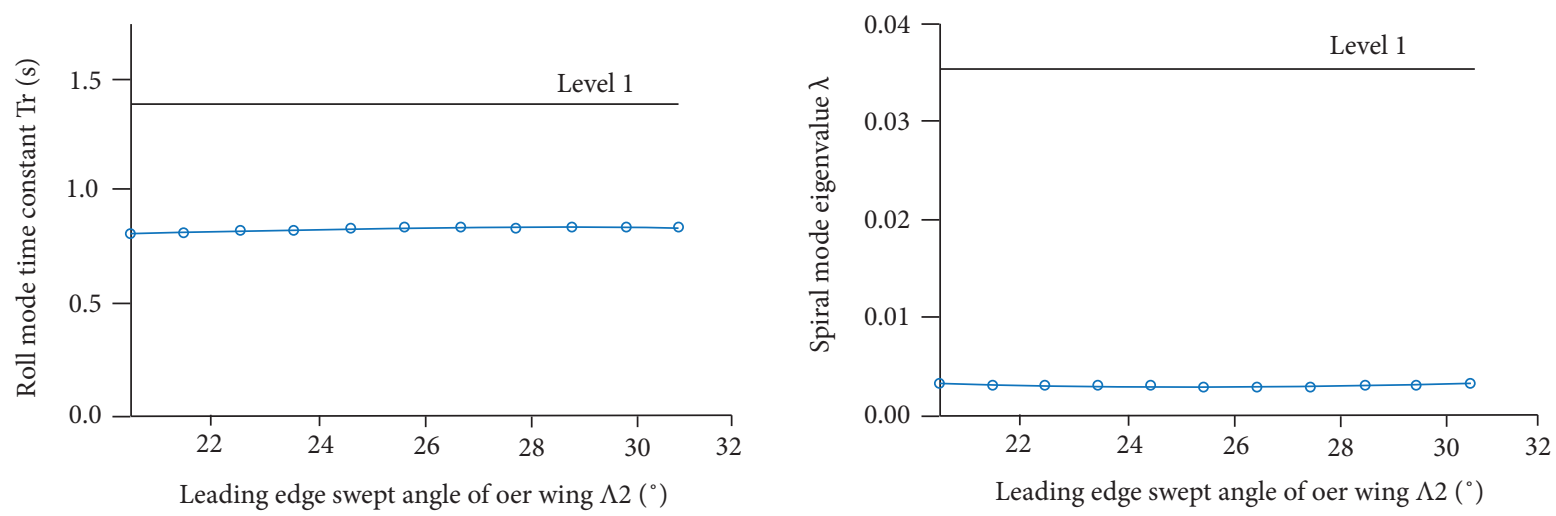

Figure 6. Influence of $\Lambda 2$ on roll mode and spiral. 


\section{IMPACT OF $\Phi$ ON DYNAMIC STABILITY}

The sensitivity of the lateral-directional stability to the changes in angle of torsion $(\Phi)$ is displayed in Table 6 and Fig. 7. The outer wing of the aircraft with linear torsion, the angle of torsion along the span, is proportional to the distance to the outer wing root. The calculation results suggest that $\Phi$ has a significant impact on lift coefficient, but little impact on roll mode and spiral mode. The lift coefficient increases with the increases of $\Phi$, further decreasing the level-flight angle of attack. The values of $\operatorname{Tr}$ and $t_{2}$ change little with the variation of the angle of torsion. Although the value of damping ratio for the Dutch roll mode obviously changes, the Dutch roll mode is divergent in the design space of $\Phi$.

Table 6. Influence of $\Phi$ on lateral-directional stability.

\begin{tabular}{|c|c|c|c|c|c|}
\hline \multirow{2}{*}{$\begin{array}{c}\text { Values } \\
{[\Phi]}\end{array}$} & \multirow{2}{*}{ AOA (deg] } & Roll mode & \multicolumn{2}{|c|}{ Dutch roll mode } & \multicolumn{1}{c|}{ Spiral mode } \\
\cline { 3 - 6 } & & $\boldsymbol{T}$ [s] & Eigenvalue & $\xi$ \\
\hline-5 & 4.6294 & 0.8509 & $0.0054 \pm 0.2961 \mathrm{i}$ & -0.0182 & 246.0592 \\
\hline-4 & 4.2829 & 0.8470 & $0.0085 \pm 0.3078 \mathrm{i}$ & -0.0275 & 244.2026 \\
\hline-3 & 3.9364 & 0.8429 & $0.0117 \pm 0.3249 \mathrm{i}$ & -0.0359 & 238.0407 \\
\hline-2 & 3.5907 & 0.8384 & $0.0151 \pm 0.3375 \mathrm{i}$ & -0.0446 & 243.9806 \\
\hline-1 & 3.2450 & 0.8339 & $0.0186 \pm 0.3547 \mathrm{i}$ & -0.0523 & 239.5330 \\
\hline 0 & 2.8994 & 0.8291 & $0.0221 \pm 0.3734 \mathrm{i}$ & -0.0591 & 239.4916 \\
\hline 1 & 2.5530 & 0.8242 & $0.0258 \pm 0.3904 \mathrm{i}$ & -0.0659 & 239.1505 \\
\hline 2 & 2.2068 & 0.8193 & $0.0294 \pm 0.4082 \mathrm{i}$ & -0.0719 & 238.9865 \\
\hline 3 & 1.8600 & 0.8142 & $0.0332 \pm 0.4275 \mathrm{i}$ & -0.0775 & 236.2356 \\
\hline 5 & 1.5129 & 0.8089 & $0.0370 \pm 0.4475 \mathrm{i}$ & -0.0824 & 233.8430 \\
\hline
\end{tabular}
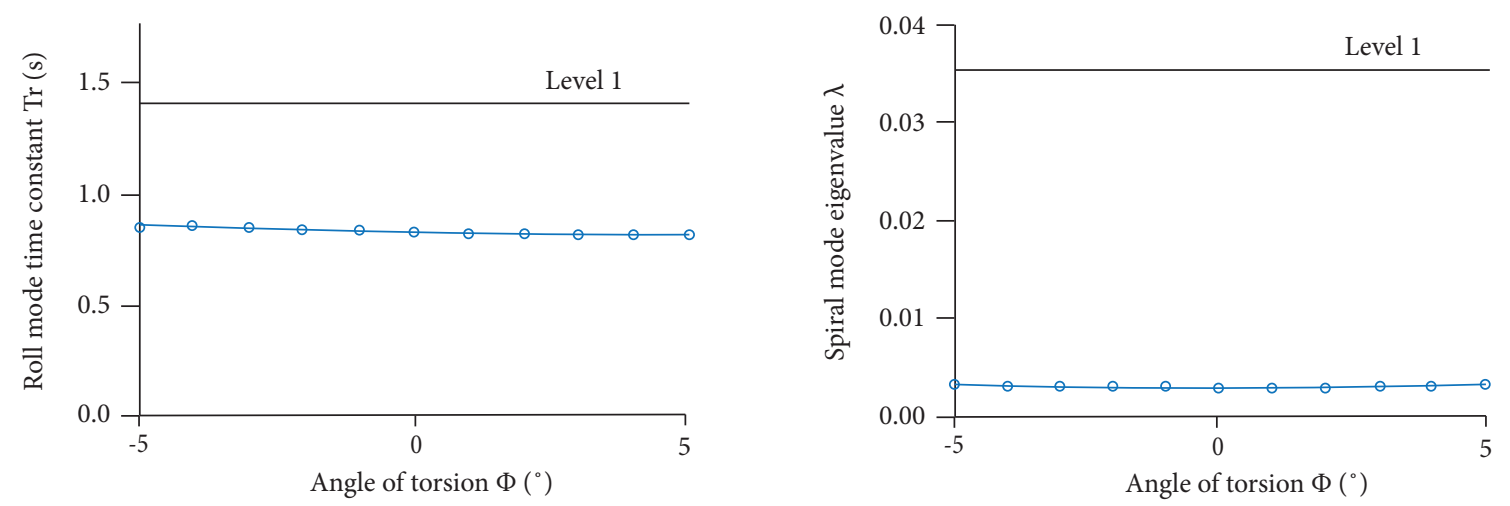

Figure 7. Influence of $\Phi$ on roll mode and spiral model

\section{IMPACT OF $\Gamma$ ON DYNAMIC STABILITY}

The variation in lateral-directional stability parameters caused by the dihedral angle $(\Gamma)$ is shown in Table 7 and Fig. 8. The results reveal that the dihedral angle has little impact on lift coefficient and roll mode, but great impact on the stability of Dutch roll mode and spiral mode. Although increasing the value of $\Gamma$ can slightly improve the rolling mode, it is obviously disadvantageous for the Dutch roll mode. In other words, the value of the damp ratio increases with the decreases of $\Gamma$. When the dihedral angle is negative, the damping ratio corresponding to the design is positive. Thus, reducing the value of the dihedral angle is useful for increasing the stability of the Dutch roll mode, which making the stability characteristic of the mode transform from divergence 
to convergence. The attendant adverse influence is that the time to double the amplitude of the spiral mode is obviously reduced. In spite of this, the spiral mode still meets the requirements of Level 1 flying quality. So the lateral-directional dynamic stability could be improved by adjusting the dihedral angle along spanwise, slightly changing the lift coefficient.

Table 7. Influence of $\Gamma$ on lateral-directional dynamic stability.

\begin{tabular}{|c|c|c|c|c|c|}
\hline \multirow{2}{*}{$\begin{array}{c}\text { Values } \\
{[\Gamma]}\end{array}$} & \multirow{2}{*}{ AOA [deg] } & \multirow{2}{*}{$\begin{array}{c}\text { Roll mode } \\
\operatorname{Tr}[\mathrm{s}]\end{array}$} & \multicolumn{2}{|c|}{ Dutch roll mode } & \multirow{2}{*}{$\frac{\text { Spiral mode }}{\text { t2 [s] }}$} \\
\hline & & & Eigenvalue & $\xi$ & \\
\hline-5 & 2.9105 & 0.9896 & $-0.0939 \pm 0.3304$ & 0.2733 & 56.5842 \\
\hline-4 & 2.9068 & 0.9331 & $-0.0579 \pm 0.3390$ & 0.1683 & 70.0947 \\
\hline-3 & 2.9043 & 0.8935 & $-0.0297 \pm 0.3466$ & 0.0853 & 89.3154 \\
\hline-2 & 2.9019 & 0.8651 & $-0.0076 \pm 0.3543$ & 0.0215 & 117.7008 \\
\hline-1 & 2.9006 & 0.8443 & $0.0095 \pm 0.3632$ & -0.0261 & 162.6411 \\
\hline 0 & 2.8994 & 0.8291 & $0.0221 \pm 0.3734$ & -0.0591 & 239.4916 \\
\hline 1 & 2.8982 & 0.8189 & $0.0307 \pm 0.3819$ & -0.0802 & 400.9449 \\
\hline 2 & 2.8982 & 0.8126 & $0.0356 \pm 0.3924$ & -0.0902 & 1096.0743 \\
\hline 3 & 2.8982 & 0.8101 & $0.0368 \pm 0.4047$ & -0.0906 & Convergent \\
\hline 4 & 2.8983 & 0.8110 & $0.0346 \pm 0.4188$ & -0.0824 & Convergent \\
\hline 5 & 2.8995 & 0.8155 & $0.0290 \pm 0.4348$ & -0.0665 & Convergent \\
\hline
\end{tabular}
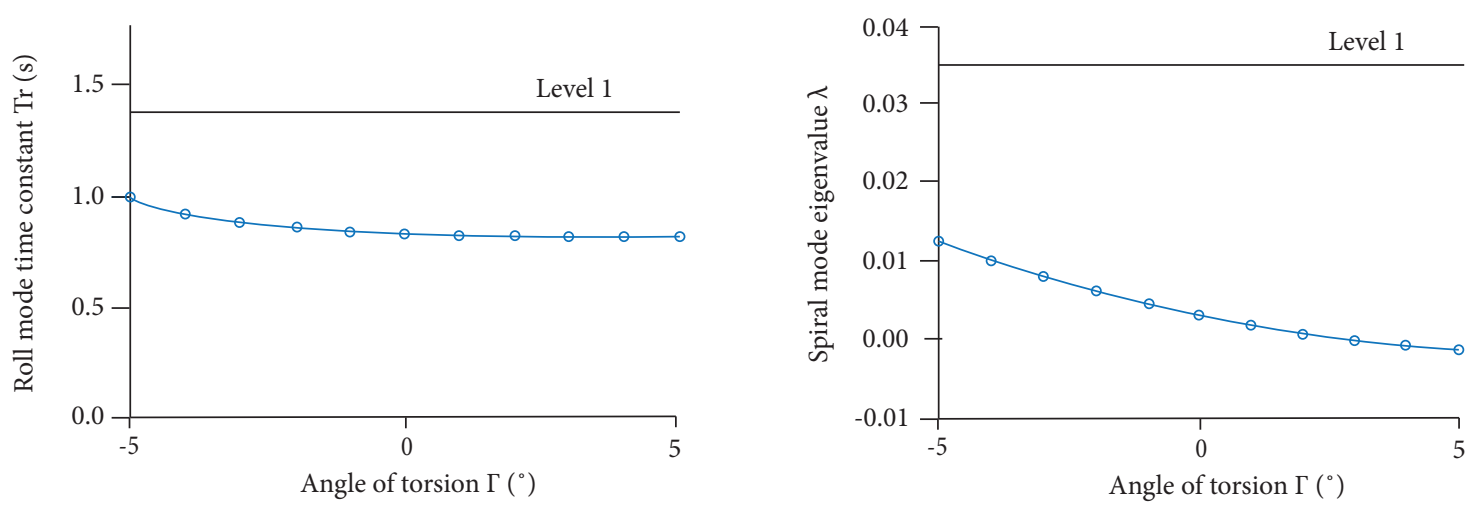

Figure 8. Influence of $\Gamma$ on roll mode and spiral model.

\section{OVERALL ANALYSIS OF THE INFLUENCE OF AERODYNAMIC SHAPE PARAMETERS}

According to the variation of assessment parameters to aerodynamic shape parameters, it can be seen that the wing aspect ratio has a relatively significant effect on the roll mode compared to other aerodynamic shape parameters, and the dihedral angle has a greater impact on the spiral modes than other parameters. However, the roll mode and spiral mode are not major factors for the flying quality of the presented UAV. Both the time constant $(T r)$ and the time to double the amplitude $\left(t_{2}\right)$ of the given schemes always meet the requirements of Level 1 flying quality. Differently from roll mode and spiral mode, nearly all the values of damping ratio $(\xi)$ are negative, which means that the Dutch roll mode of the present design is almost divergent. Under the circumstances, the flying quality of the aircraft is unacceptable and fatal. For this kind of flying wing UAV it is necessary to transform the dynamic instability into an equivalent system with good flying quality by establishing the lateral-directional stability augmentation system. 
Thus, the property of Dutch roll mode is a key factor which affects the flying quality level of lateral-directional motion modes for flying-wing configuration.

The eigenvalues of Dutch roll mode are analyzed to further investigate the impact of aerodynamic shape parameters on Dutch roll mode. Figure 9 shows the root locus of the Dutch roll mode of the present design. It can be seen from Fig. 9 that $A r$, $\Phi, \Lambda 2$ and $\Gamma$ have a significant impact on the trajectory of the eigenvalues, where $\Phi$ and $\Lambda 2$ have great influence on imaginary roots, $\Gamma$ greatly affects the real roots, and $A r$ has similar effect on both of them. As $\Phi$ and $\Lambda 2$ increase, the eigenvalue moves away from the imaginary axis rapidly, implying that the oscillation frequency of the Dutch roll mode is getting higher and higher. Reducing the value of $\Gamma$ can make the eigenvalue move from the right side of imaginary axis to the left side and away from the imaginary axis. The result demonstrates that the Dutch roll mode is transformed from divergence to convergence. Ar not only affects the oscillation frequency of the Dutch roll mode, but also influences the convergence property of the mode. In flying wing aircraft design it is possible to make the Dutch roll mode convergent by adjusting the values of wing aspect ratio, dihedral angle, and torsion angle. That is, the proper aerodynamic shape parameters also play a role as vertical stabilizers to improve the lateral-directional flying quality for flying wing aircraft.

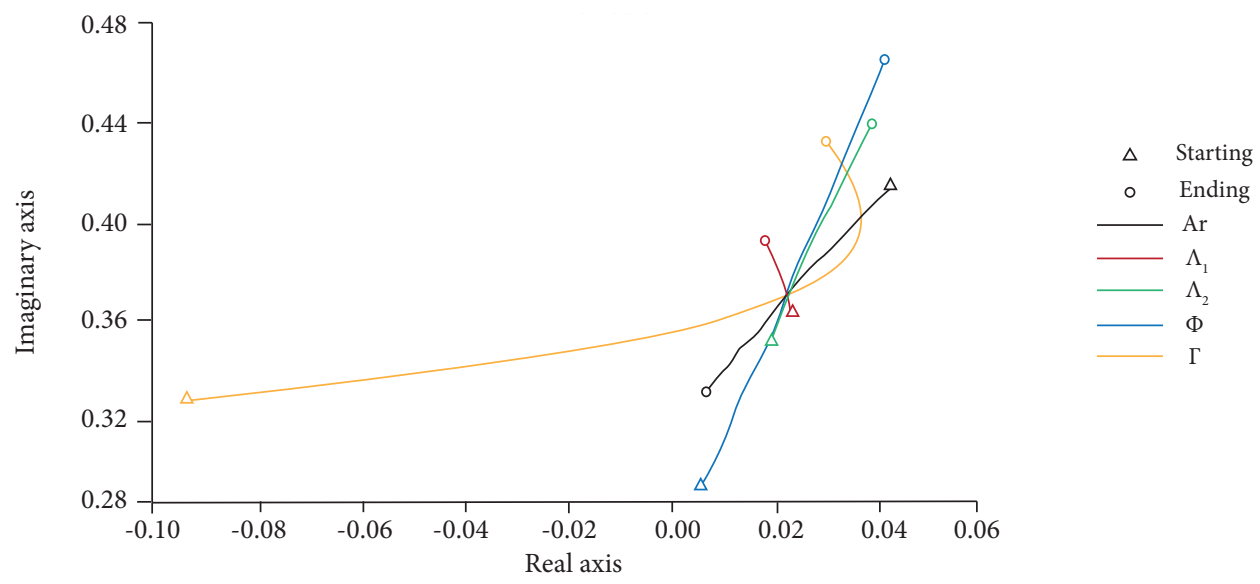

Figure 9. Root locus of the Dutch roll mode.

\section{CONCLUSIONS}

This paper studied how the aerodynamic shape parameters affect the lateral-directional dynamic stability of flying wing aircraft. The results show that the dynamic stability of the roll mode and spiral mode is better than that of the Dutch roll mode. The wing aspect ratio has a relatively significant effect on the roll mode, and the dihedral angle has a greater impact on the spiral mode than other parameters. However, the variation of the parameters does not reduce the flying quality level. Compared with the roll mode and spiral mode, the Dutch roll mode plays a more important role in the flying quality of flying-wing configuration due to the mode is always divergent. The research found that wing aspect ratio, dihedral angle, and torsion angle have an obvious influence on the convergence characteristic of the mode, and the Dutch roll mode could be converged by adjusting these parameters. So a great attention should be paid on these parameters while improving the lateral-directional dynamic stability. This paper only analyzed the lateral dynamic stability corresponding to a certain flight speed. In following research, the dynamic stability throughout the flight envelope will be analyzed.

\section{AUTHORS' CONTRIBUTION}

Conceptualization, Pan YL and Huang J; Methodology, Pan YL Investigation, Pan YL and Huang J; conceived and designed the study; Writing - Original Draft, Pan YL; Writing - Review and Editing, Huang J. 


\section{FUNDING}

There are no funders to report for this submission.

\section{REFERENCES}

Alsahlan AA, Rahulan T (2017) Aerofoil design for unmanned high-altitude aft-swept flying wings. Journal of Aerospace Technology and Management 9(3):335-345. https://doi.org/10.5028/jatm.v9i3.838

Bolsunovsky AL, Buzoverya NP, Chernyshev IL, Gurevich BI, Tsyganov AP (2014) Arrangement and aerodynamic studies for long-range aircraft in "Flying wing" layout. Presented at: 29th Congress of the International Council of the Aeronautical Sciences; Petersburg, Russia.

Bolsunovsky AL, Buzoverya NP, Gurevich BI, Denisov VE, Dunaevsky Al, Shkadov LM, Sonin OV, Udzhuhu AJ, Zhurihin, JP (2001) Flying wing—problems and decisions. Aircraft design 4(4):193-219. https://doi.org/10.1016/S1369-8869(01)00005-2

Cummings RM, Liersch CM, Schütte A, Huber KC (2016) Aerodynamics and conceptual design studies on an unmanned combat aerial vehicle configuration. Journal of Aircraft 55(2):454-474. https://doi.org/10.2514/1.C033808

Cummings R, Petterson K, Jirasek A, Schmidt S (2010) SACCON static and dynamic motion flow physics simulation using cobalt. Presented at: 28th AIAA Applied Aerodynamics Conference; Chicago, USA. https://doi.org/10.2514/6.2010-4691

Davidson R (2004) Flight control design and test of the joint unmanned combat air system (J-UCAS) X-45A. Presented at: AIAA 3rd "Unmanned Unlimited" Technical Conference, Workshop and Exhibit; Chicago, USA. https://doi.org/10.2514/6.2004-6557

Department of Defense (1980) Military specification: flying qualities of piloted airplanes. (MIL-F-8785C). US Air force Technical report. Fang ZP, Chen WC, Zhang SG (2005) Flight dynamics of aerial vehicle. Beijing: Beihang University Press.

Grellmann H (1990) B-2 aerodynamic design. In Aerospace Engineering Conference and Show; Los Angeles, USA. https://doi. org/10.2514/6.1990-1802

Liersch M, Huber KC (2014) Conceptual design and aerodynamic analyses of a generic UCAV configuration. Presented at: 32nd AIAA Applied Aerodynamics Conference; Atlanta, USA. https://doi.org/10.2514/6.2014-2001

Martinez-Val R, Schoep E (2000) Flying wing versus conventional transport airplane: the 300 seat case. Presented at: 22nd ICAS Congress; Harrogate, UK.

Melin T (2000) A vortex lattice MATLAB implementation for linear aerodynamic wing applications (Master's Thesis). Stockholm: Royal Institute of Technology.

Mialon B, Khrabrov A, Khelil SB, Huebner A, Da Ronch A, Badcock K, Cavagna L, Eliasson P, Zhang M, Ricci S, Jouhaud, JC, Rogé G. Hitzel S, Lahuta M (2011) Validation of numerical prediction of dynamic derivatives: The DLR-F12 and the Transcruiser test cases. Progress in Aerospace Sciences 47(8):674-694. https://doi.org/10.1016/j. paerosci.2011.08.010

Pan Y, Huang J, Li F, Yan C (2017) Application of Multidisciplinary Design Optimization on Advanced Configuration Aircraft. Journal of Aerospace Technology and Management 9(1):63-70. https://doi.org/10.5028/jatm.v8i4.736

Petterson K (2006) CFD analysis of the low-speed aerodynamic characteristics of a UCAV. Presented at: 44th AIAA Aerospace Sciences Meeting and Exhibit; Reno, USA. https://doi.org/10.2514/6.2006-1259

Saeed T, Graham W, Babinsky H, Eastwood J, Hall C, Jarrett J, Lone MM, Seffen K (2009) Conceptual design for a laminar flying wing aircraft. Presented at: 27th AIAA Applied Aerodynamics Conference; San Antonio, USA. https://doi.org/10.2514/6.2009-3616

Schütte A, Huber KC, Boelens OJ (2014) Static and dynamic numerical simulations of a generic UCAV configuration with and without control devices. Presented at: 32nd AIAA Applied Aerodynamics Conference; Atlanta, USA. https://doi.org/10.2514/6.2014-2132

Snyder MP, Weisshaar TA (2013) Flutter and directional stability of aircraft with wing-tip fins: conflicts and compromises. Journal of Aircraft 50(2):615-625. https://doi.org/10.2514/1.C031978

Song L, Yang H, Xie JF, Huang J (2014) Predicting stability derivatives of flying wing aircraft based on improved vortex lattice method. Journal of Nanjing University of Aeronautics \& Astronautics 46(3):457-463.

Song L, Yang H, Yan X, Ma C, Huang J (2015) A study of instability in a miniature flying-wing aircraft in high-speed taxi. Chinese Journal of Aeronautics 28(3):749-756. https://doi.org/10.1016/j.cja.2015.04.001

Vicroy DD, Loeser TD, Schuette A (2010) SACCON forced oscillation tests at DNW-NWB and NASA Langley 14X22-foot tunnel. Presented at: 28th AIAA Applied Aerodynamics Conference; Chicago, USA. https://doi.org/10.2514/6.2010-4394 
Whittenbury J (2011) Configuration design development of the navy UCAS-D X-47B. Presented at: AIAA Centennial of Naval Aviation Forum "100 Years of Achievement and Progress"; Virginia Beach, USA. https://doi.org/10.2514/6.2011-7041

Zhang M, Rizzi A, Meng P, Nangia R, Amiree R, Amoignon O (2012a) Aerodynamic design considerations and shape optimization of flying wings in transonic flight. Presented at: 12th AIAA Aviation Technology, Integration, and Operations (ATIO) Conference and 14th AIAA/ISSMO Multidisciplinary Analysis and Optimization Conference; Indianapolis, USA. https://doi.org/10.2514/6.2012-5402

Zhang ZJ, Wang L, Wang LX, Wang JJ (2012b) Three-axis stability characteristics of flying wing with high aspect ratio. Systems Engineering-Theory \& Practice 5(32):1129-1135.

Zhou HX, Liu B (2015) Characteristics analysis and optimization of flying-wing vehicle structure. Advanced Materials Research 1077:177-184. https://doi.org/10.4028/www. scientific.net/AMR.1077.177 